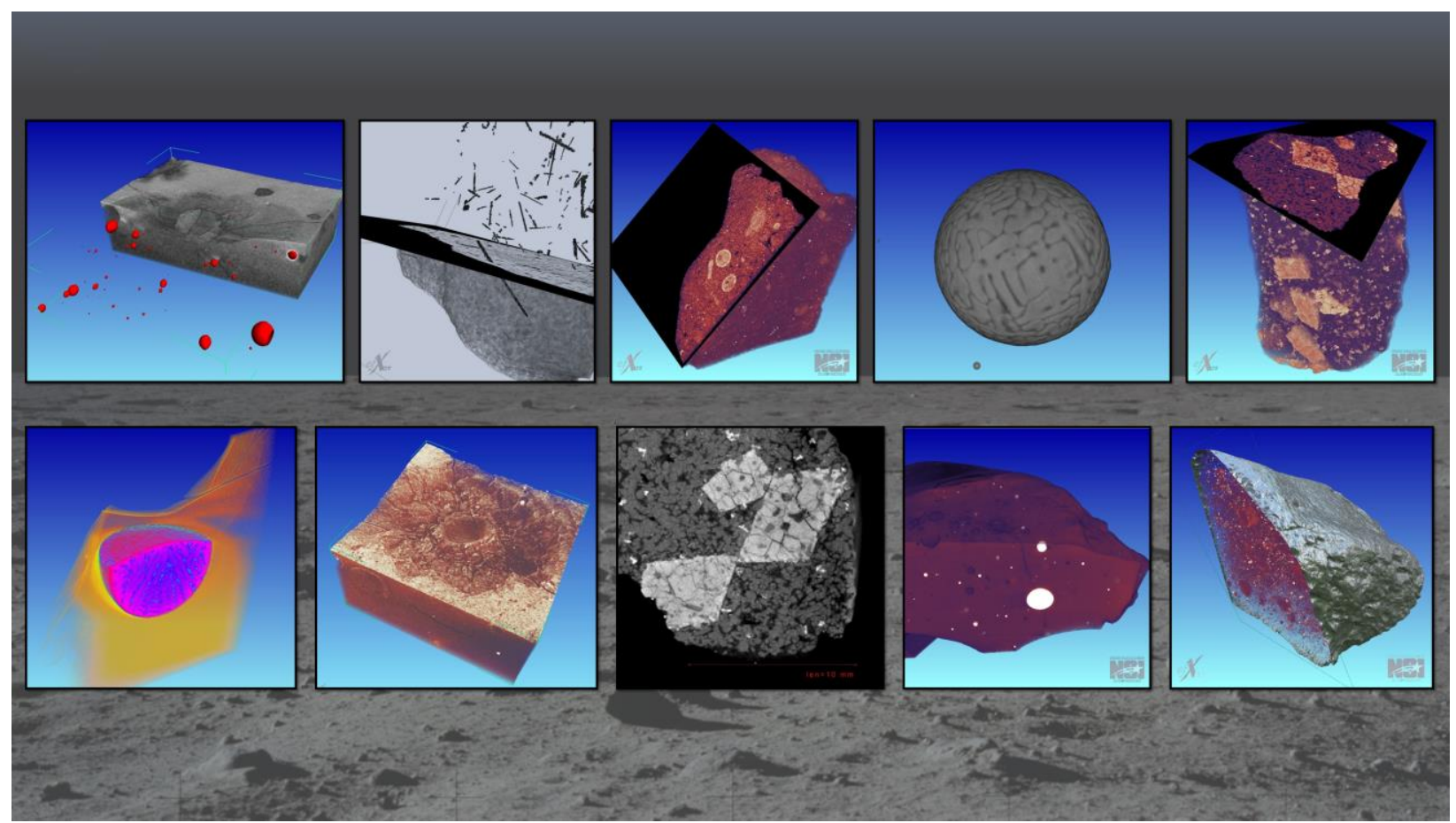

\title{
Lunar Exploration in 3D: Volumetric Imaging of Lunar Materials and Manufactured Materials via x-ray Computer Tomography
}

Authors: James B. Garvin \& Justin S. Jones (NASA Goddard, Greenbelt MD 20771)

\section{Co-authors:}

James W. Head III (Brown University), Amy McAdam (GSFC), Noah Petro (GSFC), Kelsey Young (GSFC), Ryan A. Kent (GSFC), Nathan A. Smith (GSFC)

\section{Co-signees:}

John Grunsfeld (NASA Astronaut), Barbara Cohen (GSFC), Paul Mahaffy (GSFC), Michael Amato (GSFC), George Studor (NASA/NESC), Tracie Prater (MSFC), Lawrence Huebner (MSFC), William Prosser (LaRC/NESC), Juliane Gross (JSC)

Submitted to: National Academy of Sciences Planetary Sciences and Astrobiology Decadal Survey as a White Paper 
SUMMARY: Volumetric x-ray imaging of lunar surface materials, employing rapidly-advancing, quantitative characterization methods, is well-suited as a new "tool" in NASA's return to the lunar surface via Artemis (Space Policy Directive-1) and amply justified as a means of characterizing, inspecting, and assessing critical parameters for effective human exploration. Apollo samples of lunar materials (dust, regolith, lithic fragments, clasts) and subsequent studies associated with crew safety, plasma dynamics, and micro-meteorite cratering [1-4] have painted a picture of an incompletely characterized "system" of particular importance as science investigations are integrated with human exploration and commercial activities over the next decade. Nondestructive evaluation (NDE) methods such as micro-focus x-ray Computed Tomography (xCT) can now be used to evaluate such lunar materials ranging in size from roughly $1 \mathrm{~mm}$ to $100 \mathrm{~mm}$ and larger [5-7, 15, 19], providing new engineering boundary conditions for programs such as Artemis. While these studies have recently been limited to terrestrial laboratories, extending this capability to space will enable new possibilities for use on the Moon including in-space manufacturing (ISM), in-space resource utilization (ISRU), astrobiology of lunar materials, and models of the micro-cratering processes relevant across the solar system [6, 8]. A critical first step is implementation and utilization of the first in situ volumetric imaging via $\mathrm{XCT}$ on the Moon, which would open the door to other complimentary techniques and a new dimension of understanding. Engineering-relevant evaluation via $\mathrm{xCT}$ of in situ materials as well as those that will be additively manufactured on the Moon is essential as women and men return to deep space. Here we emphasize the potentially transformational opportunities associated with operation of scalable xCT imaging systems on the Moon in support of exploration and the applied science and engineering that comes from human activities in the next decade.

\section{INTRODUCTION:}

Micro-focus x-ray Computer Tomography (xCT), Laser Confocal Microscopy (LCM), Scanning Electron Microscopy (SEM), as well as other next-generation tools (XPS, XRF, photogrammetry), are now part of an expanded arsenal of NDE capabilities that are poised to explore lunar materials in the era of Artemis, using the legacy Apollo samples [5, 6, 15, 24-26] and extending to lunar materials sampled over the next decade by new partnerships in human spaceflight, commercial interests, and international players.

Many lunar materials remain minimally characterized, and yet when analyzed could answer engineering challenges recognized for decades by science pioneers such as Dave McKay, Larry Taylor, and many others [1-3, 9-21, 25]. Understanding this integrated system of lunar materials couples the physics of micro-cratering, space weathering, plasma dynamics, and geochemistry in an ensemble of processes that produce phenomena that vary on the Moon as a function of location, environment, and dynamics $[12,18,19,21]$. X-ray CT offers a detailed, holistic perspective of these various processes, giving a view that quite literally paints a picture of the entire interconnected system. As Artemis moves forward, we recommend strong attention on utilization of NDE techniques for characterizing the interiors of lunar surface materials down to the finest possible scales $[3,15,20,24]$ with potential measurement opportunities on the lunar surface, on the cis-lunar Gateway, and in laboratories and curation facilities on Earth (e.g., NASA JSC [5, 12]). In particular, the application of $x C T$ volumetric imaging measurements (Figs. 1-3) will address key variables and unknowns associated with exploration-relevant lunar materials [1, 10, 21, 25-28] that represent aspects of multiple strategic knowledge gaps (SKG's) for the Moon [3]. 



Figure 2: CAPTEM-approved Apollo 16 sample number 60095, which consists of a centimeter long fragment of a spherical lunar impact melt glass. Left image shows $\sim 1 \mathrm{~mm}$ wide section centered on a micrometeorite impact crater, which has been cross-sectioned virtually in the XCT volumetric imaging data to reveal an array of interior metal filled vesicles, or vugs (red). Right image shows a close-up of one of these particles, rescanned under ultra-high resolution using a Zeiss Xradia system at the US Army Research Lab (courtesy US ARL, Aberdeen Materials Science facility, via Dr. J. Sietins and others).

Crew-based measurements and experiments of in situ materials represent an opportunity for advancing understanding of deep space processes, environments, and engineering/safety challenges that will support the sustainability of human exploration on the Moon and eventually on to Mars. Especially important are precursor measurements of the interior or volumetric properties of representative lunar materials that today require laboratory preparation or relatively large xCT equipment. Such volumetric imaging accomplished on the Moon will offer in situ capabilities that today are only possible in terrestrial laboratories, enabling everything from sample triage for resources [25] to new scientific discoveries. Additionally, such measurement capabilities will facilitate inspection of ISM parts developed on the Moon from lunar materials, enabling their effective utilization for exploration and also for potential commercial interests. 


\section{BACKGROUND:}

High precision volumetric measurements of the interior 3D shape and composition [19] of lunar regolith particles are largely unavailable (e.g., [4] quantified dust), and yet this aspect of the lunar surface environment, and its association with regolith formation and evolution [11], as well as future utilization of the Moon, presents a fundamental engineering boundary condition that connects science (from Apollo samples) to National Space Council policy objectives in SPD-1. In addition, the importance of lunar fines has been emphasized in multiple science prioritization documents [e.g., Visions and Voyages, 2011 - NASEM Planetary Decadal Survey], as well as via investments extending from the LADEE mission [10], LRO, and lunar simulants [2, 3]. While micro-focus x-ray CT may not be the optimal approach for characterizing dust $(<100 \mu \mathrm{m}$ in diameter) in terms of individual particles, it will be impactful when used as a complimentary tool to assess packing densities in drill cores, provide particle size distributions for larger $(\sim 100 \mu \mathrm{m}$ and larger) particles, and provide relative density characterization. Here we suggest that engineering-quality measurements of Apollo samples with emphasis on 3D volumetric analysis, using available NASA Non-Destructive Evaluation (NDE) methods [5-7, 25], in combination with advanced data analysis software (including Machine Learning and Neural Networks for smart data reduction and/or automated feature detection), is an amply warranted step forward to eliminate key unknowns as we return to the Moon via Artemis. Such integrated NDE-based measurements (especially xCT) would serve as a pathfinder for studies of the origin, evolution, and significance of materials on the Moon [15] and on other solar system airless body surfaces, together with their connection to micro-cratering processes on such objects $[8,11]$. Results will have a bearing on new samples soon to be returned from the asteroid Веnпи by OSIRIS-REx [23], as well as for refining engineering solutions for upcoming human exploration activities at the lunar surface (such as Artemis-3, VIPER rover, and other landed systems via CLPS).

The Apollo missions acquired samples that include varieties of the lunar dust, agglutinates and lithic fragments, as demonstrated in the published measurements [1-4, 15, 21]. Via this White Paper, we recommend pathfinding and foundational measurements that will provide the lunar science and engineering community with quantitative knowledge that extends the existing (but limited) measurements in the Lunar Sourcebook [12, 18] and other key references in support of Artemis. Using state-of-the-art micro-focus xCT methods and newlyavailable segmentation and Machine Learning (ML) software for volumetric data analysis, we suggest that cataloging new aspects of the Moon (i.e., from $\sim 500 \mu \mathrm{m}$ or larger dust to clasts the size of baseballs) will catalyze additional community-based investigations and more exhaustive crew-based measurements going forward that will contribute to ISM, crew safety, sustainability (ISRU), and potentially to astrobiology. There is a scientific and engineering urgency to characterize the volumetric properties of lunar materials with emphasis on key regolith components [4, 15], but also on micro-craters and other diagnostic features smaller than $\sim 10 \mathrm{~cm}$ $[8,19]$. Recently, CAPTEM-approved analyses of a number of Apollo 16 samples (e.g., 60095,36) containing micro-craters has been undertaken using xCT and related methods at GSFC (via the Code 541 NDE facility [6]); Figures 1-3 illustrate example results. Connecting these surveys of the interior geometric properties of lunar micro-craters [8] to new measurements of regolith materials (Fig. 2) is another critical objective of the recommended volumetric measurement campaign on the Moon. 


\section{METHODS: NDE goes to the Moon}

Circa 2020, a suite of NDE measurement capabilities are now well-established in NASA and industrial laboratories for materials sciences, engineering, and applied geosciences [7]. These include Laser Confocal Microscopy (LCM) for defining 3D properties of materials down to tens of nanometers (vertical), SEM/TEM imaging for morphologies down to $<10 \mathrm{~nm}$, and x-ray Computed Tomography (xCT) for particles $\sim 500 \mu \mathrm{m}$ or larger. Application of these and other more "destructive" methods for characterizing lunar materials are now possible via several facilities across NASA. Surface-deployed NDE measurement systems, including xCT scanners for AM parts screening, safety, inspection, and sample triage (Fig. 4) have the potential to contribute to fundamental new measurements at the Moon, which themselves will inform future approaches as sustained human presence is established [6,7]. Efforts are underway at NASA to develop pathfinder instruments for some of these techniques for space (e.g., proto-xCT, nicknamed "iVOXEL": Fig. 4) which will benefit from new analyses of Apollo soil samples over the next few years. For example, an integrated xCT examination of Apollo 16 highlands soils documents scales of features related to the sources and sinks of lunar dust, from micro-craters (Fig. 2) to dust affixed to lunar glasses and agglutinates (Fig. 3).



A micro-focus xCT system on the Moon (Fig. 4) would offer several advancements beyond the current state of the art. First, it provides the ability to non-destructively interrogate the interior of the object, via either virtual "cut planes" or dynamic 3D visualization tools (flythrough videos, translucent images, customizable section views, virtual reality visualization, etc.). It also offers unique ability to measure internal features and sample dimensions, including more advanced measurements such as volumetric particle size distribution, internal surface areas, and more. When combined with other, complementary tools such as photogrammetry methods (for example, digital image correlation (DIC or VIC) and structure from motion (SfM)) or analytical tools (such as laser induced breakdown spectroscopy (LIBS) and x-ray fluorescence 
(XRD)), the 3D mapping capabilities of xCT helps provide much needed internal context and understanding of volumetric distributions. This is a new step for materials characterization and inspection well-suited for Artemis as humans conduct operations on the lunar surface.

\section{EXAMPLES OF OPPORTUNITIES}

NASA's prime directive and the related Artemis program calls for return of human explorers (women \& men) to the Moon in less than a decade, with an armada of commercial, international, and NASA robotic precursors between 2021 and 2025. Commercial Lunar Payload Services (CLPS) landers will carry a first-wave of payloads to evaluate the Moon and the VIPER lunar resources rover will address materials characterization for ISRU [26]. When Artemis-3 lands women on the Moon, it could be equipped with simple instrumentation for measuring the lunar regolith, which is often the most difficult to handle after return to Earth laboratories [4]. New technologies for lunar materials characterization are now utilized via industrial laboratories and some of these approaches, including micro-focus xCT, can readily be adapted for the Moon [5-7].

The suite of accessible lunar regolith materials in general defines new opportunities for in situ experimentation, building on the work by multiple NASA-funded virtual institute (SSERVI) teams over the past decade (e.g., DREAM-1,2). One compelling concept involves the so-called astrobiology of the Moon and the consideration of agnostic life detection principles, as recently established by Professor Sarah Johnson's Laboratory for Agnostic Bio-signatures (LAB), which is a Research Coordination Network within NASA's Astrobiology program [27-28]. It is possible that microbiological experimentation on lunar fine materials can form the basis of a new line of research as biological systems are sent to the Moon for long-term, sustainable activities that include living systems at many scales. Other habitability-related aspects of lunar materials may also be relevant, as in [1]. In all of these cases, having volumetric, 3D interior models of relevant materials will advance science and engineering as creative experiments are conducted [28].

Volumetric imaging tools such as $\mathrm{xCT}$ offer the key to understanding true 3D (interior) interrelationships within samples (Fig. 1), such as void tortuosity, crack propagation, through thickness texture, compositional distribution, and more. Being able to rely on full volumes, rather than just a glimpse through destructive, single plane slices, offers unrivaled documentation of spatial and size distributions of vesicles, particles, lunar fines, or inclusions. This approach not only has the potential to significantly increase measurement fidelity of features within a sample, but it also is a crucial tool when used as a sort of 3D map in concert with other complementary diagnostic tools such as SEM, LCM, or geochemical analysis. Knowing the composition or other relevant detail from surface measurements, but then being able to extend that knowledge into the depth of the sample beyond the limits of the surface analysis by relational mapping/co-registration with the xCT data, offers truly next-generation capability. That we could be on the verge of accomplishing this volumetric sample reconnaissance remotely will greatly enhance the quality of future sample return missions by enabling in situ, high-grade scientific triage. In addition, this new family of deployable instruments will dramatically enhance crew-based science operations on the lunar surface, especially when used in concert with other compact tools (LIBS, XPS, etc.).

\section{RECOMMENDATIONS}

Here we suggest that all of the science goals and objectives outlined above can be accomplished by embracing a new family of volumetric imaging tools associated with xCT. Recent concept

studies at GSFC have demonstrated that the Size, Weight, and Power (SWaP) of an associated 
$\mathrm{xCT}$ system in space, as well as operations and data management (possibly with support from ML tools), is viable. Exactly what these near-term xCT systems will evolve to be depends on mission scope and application. A typical xCT scanner consists, most basically, of an x-ray source, an x-ray detector, and a mechanism for manipulating the sample in between these sub-systems (e.g., for industrial xCT this is generally a motorized turntable). The required hardware geometry is flexible and scalable to different size regimes, so in theory a whole portfolio of in space xCT systems could be developed for different applications. Advancing technical readiness through a first flight demonstration (potentially an ISS pathfinder instrument) will be key for flight-proving the concept, at which point the technology could then be tailored on the basis of application/destination. Ultimately, xCT concept refinement will depend in on the relevant sample size and materials of interest, but the fabrication of the hardware involves no unsurmountable challenges beyond those typical of building new flight instruments. For example, an autonomous XCT scanner on a future Mars mission might utilize a robotic arm to provide both sample retrieval and sample manipulation within the scanning system (Fig. 4). A crew-tended xCT within a lunar habitat may not require the complication of such a robotic arm, but could take advantage of its relative simplicity by offering a larger, open-platform scan volume and make use of higher power sources (for larger, more dense objects). This could enable a multi-physics instrument whereby xCT works in tandem with those aforementioned complementary tools, such as a photogrammetry camera system that maps surface coordinates (while the CT scan is running) and perhaps a LIBS tool that can then target a location on the surface that has been co-registered with the surface and volume datasets.

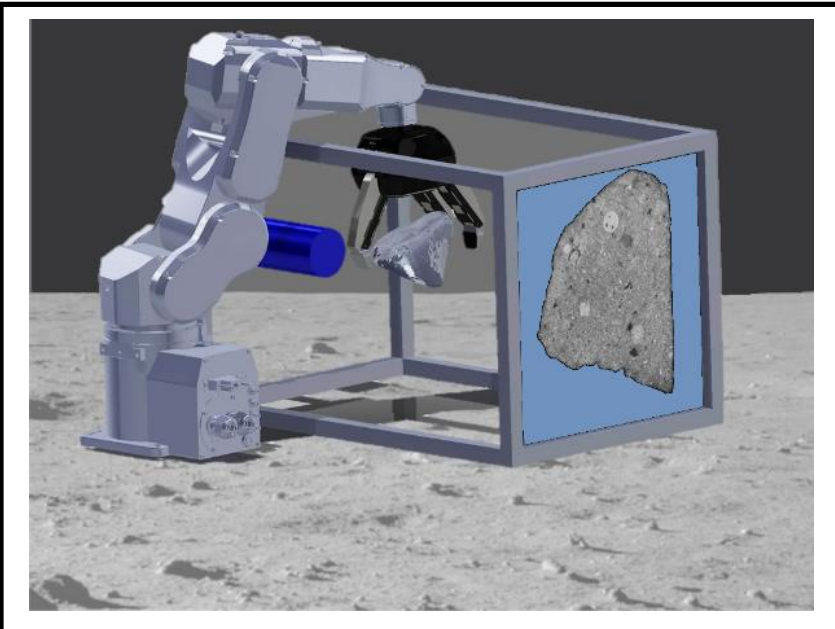

Figure 4: Possible future design of an XCT scanner to support Artemis, showing an integrated 6 DOF robotic arm capable of sample capture and articulation (artist concept of CT process shown). Such a volumetric scanning system would provide high-resolution non-destructive views of lunar materials [6]. This concept, nicknamed iVOXEL ("i" Volumetric Omni-purpose X-ray Evaluation Laboratory), could also be implemented on early Artemis surface missions via a "suitcase" deployment system in which crew members carry and deploy the XCT on the surface and "feed it" samples while conducting other EVA activities.

\section{APPLICATIONS}

The suite of lunar surface materials presents a scientific opportunity that is associated with overarching safety and engineering considerations as human/robotic systems are established on the Moon in the near term [1-4]. While in many ways lunar regolith materials must be mitigated as a hazard, they also must be evaluated as a long-standing part of the lunar environment $[1,10]$ and regolith sedimentary systems [11], with interactions that may relate to the lunar water cycle, dusty plasma dynamics, and space weathering [10,15,19,21]. Volumetric characterization of the sometimes neglected lunar regolith system is nonetheless important and significant as science learns from the Moon in new ways in order to establish paradigms that are also relevant to asteroids and eventually to Mars. Applying NDE measurement systems (xCT) to existing Apollo regolith samples and extending them to new samples both on the Moon and those returned to Earth can be 
accomplished within the framework of safety, inspection, resources utilization, and applied science (geotechnical). Innovative development of in space xCT systems represents a key step, with benefits across multiple disciplines [5, 7, 23, 26-28]. As humans return to the lunar surface, nextgeneration measurement systems to enhance their productivity, such as the $\mathrm{xCT}$ scanners described herein are particularly relevant to the intersection of science and engineering as part of exploration.

\section{CONCLUSIONS}

The lunar surface and its broad suite of materials awaits our return via human exploration systems such as Artemis. While lunar regolith is viewed by many experts as an important issue to circumvent, it also offers a compelling set of opportunities for exploring the nearly invisible "interior" side of the lunar frontier, and one that is in a constant state of regeneration [9-12, 15, 19]. Today's laboratory-based NDE measurement systems and the highly-capable analysis software that are available for analysis makes the volumetric imaging of lunar materials an accessible problem, tied to fundamental solar system processes for which the Moon will always an ideal "natural laboratory" $[2,3,10,12,15,27]$. As such, volumetric imaging by xCT could become an essential tool (Error! Reference source not found. 3) as we sustain a presence on the Moon as humanity's first deep-space outpost, and learn to explore this critical frontier while "being there".

References Cited: [1] McKay D. S. et al. (2015) Acta Astronautica 107, p. 163-176. [2] Taylor L. et al. (2016) Planetary and Space Sci. 126, p. 1-7. [3] Taylor L. et al. (2005) AIAA $1^{\text {st }}$ Space Exploration Conference, p. 2500-2501. [4] Park J. et al. (2008) Journal of Aerospace Engineering (ASCE), vol. 21, p. 266-271. [5] Zeigler R. et al. (2014) Microscopy and Microanalysis 20 (no. 3), p. 636-637. [6] Garvin J. B. et al. (2019) LPSC 50 th \# 1708 (Houston TX). [7] Brown E. (2018) Photonics \& Imaging Technology, May1,2018; URL: $\quad$ https://www.techbriefs.com/component/content/article/tb/supplements/ pit/features/articles/28887.[8] Horz F. et al. (1975) Plan. And Space Sci. 23, 151-170. [9] O’Brien B. (2011) Planetary and Space Sc. 59, p. 1681-1690. [10] Grun E. et al. (2011) Planetary and Space Sci. 59, p. 16721680. [11] Oberbeck V. (1975) Reviews of Geophysics and Space Physics, vol. 13, p. 337-362. [12] McKay D. S. et al. (1991) in Lunar Sourcebook, Cambridge U. Press, p. 286-356. [13] Taylor L. et al. (2001) JGR 106 (E11), p. 27985-27999. [14] Liu Y. et al. (2007) Am. Mineralogist 92, p. 1420-1427. [15] Noble S. K. et al. (2001) Met. \& Plan. Sci. 36, p. 31-42. [16] Butler J. \& E. King (1974) Proc. $5^{\text {th }}$ Lunar Sci. Conf. (Vol. 1), p. 829-841. [17] Graf J. C. (1993) Lunar soils grain size catalog, NASA Ref. Pub. C-1265, Washington DC. [18] Carrier W. D. et al. (1991) in Lunar Sourcebook, Cambridge U. Press, p. 475-594. [19] Wentworth S. et al. (1999) Met. \& Plan. Sci. 34, p. 593-603. [20] Papike J. et al. (1982) Proc. $10^{\text {th }}$ Lunar \& Plan. Sci. Conf., p. 409-420. [21] Carrier D. (2003) Journal of Geotechnical \& Geoenvironmental Engr. 129, p. 956959. [22] Garvin J. B. et al. (2014) 45 th LPSC, \# 2511 (Houston, TX). [23] Lauretta D. et al. (2017) Space Science Reviews, Volume 212, Issue 1-2, p. 925-984. [24] Dukes C. A. \& R. A. Barogiola (2015) Icarus 255, p. 51-57. [25] Bradley J. P. \& Z. R. Dai (2009) MAPS 44 (10), p. 1627-1642. [26] ISRU 2019 Workshop report; https://www.hou.usra.edu/meetings/lunarisru2019/workshop-report.pdf \{from LEAG\}. [27] Johnson, S. S. et al. (2020) Astrobiology, 20(2), 167-178. doi.org/10.1089/ast.2017.1812. [28] Johnson, S. S. et al. (2020) Detecting Life on Earth and the Limits of Analogy; in Planetary Astrobiology (V. Meadows et al., eds.), pp. 121-150. University of Arizona Press.

Acknowledgments: We acknowledge the support of the lunar curators at JSC, CAPTEM, and the encouragement of GSFC senior leadership (Drs. Andrucyk, Johnson, Kinney, Hughes). Special thanks to Dr. Jennifer Sietins and colleagues (US Army ARL at Aberdeen, MD), as well as GSFC's Materials Engineering Branch. 